\title{
A NOTE ON CHARACTERS OF ALGEBRAIC GROUPS
}

\author{
S. A. BROUGHTON
}

\begin{abstract}
It is proven that a nowhere-vanishing polynomial function on a connected algebraic group, over an algebraically closed field, is a multiple of a (one-dimensional) character.
\end{abstract}

It is well known that any nowhere-vanishing polynomial function on $\mathrm{GL}_{n}(k), k$ algebraically closed, is a constant multiple of a power of the determinant. This is most easily seen by observing that the coordinate ring of $\mathrm{GL}_{n}(k)$ is $k\left[X_{i j}\right.$, $\left.\operatorname{det}^{-1}\left(X_{i j}\right)\right]$ $(i, j=1, \ldots, n)$, so any unit in this ring, i.e., a nowhere-vanishing polynomial function $f$ on $\mathrm{GL}_{n}(k)$, must be of the form $f(x)=c \cdot \operatorname{det}^{m}(x)$, where $c \in k^{*}$ and $m$ is an integer. This result is not an isolated fact about $\mathrm{GL}_{n}(k)$; it holds for algebraic groups in general.

THEOREM. Let $G$ be a connected, affine, algebraic group over the algebraically closed field $k$, which may be of any characteristic. Let $f: G \rightarrow k^{*}$ be any nowhere-vanishing polynomial function on $G$ satisfying $f(e)=1$. Then $f$ is a character, i.e., $f(x y)=$ $f(x) f(y)$ for $x, y \in G$.

This theorem was originally proven by M. Rosenlicht [R, Theorem 3] using methods which are quite different from those used here. We shall use the well-known structure theory of affine algebraic groups (cf. J. E. Humphrey's monograph [H], for example) to give an elementary proof of this theorem. The author wishes to thank the referee for pointing out the article of M. Rosenlicht.

Proof of The Theorem. We prove the Theorem in four steps by first considering unipotent groups, then tori, solvable groups and, finally, the general case.

Case 1. Unipotent groups. Since $G$ is unipotent, there is a descending chain of closed subgroups $G=G_{0} \supseteq G_{1} \supseteq \cdots \supseteq G_{s}$, such that $G_{i+1}$ is normal in $G_{i}$, and $G_{s}$ and the quotients $G_{i} / G_{i+1}, i=0, \ldots, s-1$, are one-dimensional unipotent groups. Any one-dimensional, connected, unipotent group is isomorphic to the additive group of $k$ [H, Theorem 20.5]. Therefore, $f$, restricted to $G_{s}$, is a polynomial without zeros on $k$, so it must be a constant. As $f(e)=1$ we see that $f=1$ on $G_{s}$ and is the trivial character. Each coset $x G_{s}, x \in G_{s-1}$, is isomorphic to $k$ and the above argument shows that $f$ is constant on $x G_{s}$ as well. This implies there is a polynomial function $f^{\prime} \in k\left[G_{s-1} / G_{s}\right]$ such that $f(x)=f^{\prime}(\pi(x)), x \in G_{s-1}$, where $\pi: G_{s-1} \rightarrow$ $G_{s-1} / G_{s}$ is the canonical projection. Because $G_{s-1} / G_{s}$ is isomorphic to $k, f^{\prime}$ is also a

Received by the editors May 13, 1982 and, in revised form, January 17, 1983.

1980 Mathematics Subject Classification. Primary 20G99; Secondary 16A25.

Key words and phrases. Character, affine algebraic group.

(C)1983 American Mathematical Society $0002-9939 / 83 / 0000-1448 / \$ 01.75$ 
constant, so $f$ is the trivial character on $G_{s-1}$. The remainder of the proof is a simple induction argument.

Case 2. Tori. If $G=k^{*}$, then any polynomial function on $G$ is of the form $f(x)=x^{n}\left(a_{0}+a_{1} x+\cdots+a_{m} x^{m}\right), n, m \in Z, m \geqslant 0, a_{i} \in k, a_{0}, a_{m} \neq 0$. It is clear that, unless $m=0, f(x)$ will have a zero in $k^{*}$, so $f(x)=a_{0} x^{n}$. However, $a_{0}=f(1)=1$, so $f(x)=x^{n}$, a character of $k^{*}$. For the general torus we argue by induction, so we assume the Theorem is true for any torus of dimension $n$. An $(n+1)$-dimensional torus is of the form $k^{*} \times T$, where $T$ is an $n$-dimensional torus. For $x \in k^{*}$, let $f_{x}^{\prime} \in k[T]$ be the function on $T$ defined by $f_{x}^{\prime}(t)=f(x t) / f(x)$. Clearly, $f_{x}^{\prime}(e)=1$, and no $f_{x}^{\prime}$ vanishes on $T$, so they are all characters of $T$. There is a finite-dimensional vector subspace $W$ of $k\left[k^{*} \times T\right]$ containing $f$ and invariant under the action of $k^{*} \times T$ by left translations [H, Proposition 8.6]. If $\rho: k\left[k^{*} \times T\right] \rightarrow k[T]$ is the projection given by restricting functions on $k^{*} \times T$ to $T$, then $V=\rho(W)$ is finite dimensional and contains all $f_{x}^{\prime}, x \in k^{*}$. Since characters are linearly independent, $V$ contains at most finitely many characters. The map $k^{*} \rightarrow V, x \rightarrow f_{x}^{\prime}$ is a morphism whose image is contained in a finite set and, since $k^{*}$ is connected, its image must be a single point. This means $f_{x}^{\prime}=f_{e}^{\prime}$ or $f(x t)=f(x) f(t), x \in k^{*}, t \in T$, and this readily implies that $f$ is a character since it is already a character when restricted to the subgroups $k^{*}$ and $T$.

Case 3. $G$ solvable. The group $G$ is a semidirect product $T U$, where $T$ is a maximal torus and $U$ is the unipotent radical of $G$ [H, Theorem 19.3]. By Cases 1 and 2, $f$ is a character when restricted to $T$ and is constant on the cosets $t U, t \in T$. Using these facts, along with the normality of $U$, a straightforward calculation shows that $f$ is a character.

Case 4. $G$ any affine, connected group. Let $B$ be any Borel subgroup of $G$. Again for $g \in G$ we let $f_{g}^{\prime}$ be the function on $B$ defined by $f_{g}^{\prime}(b)=f(g b) / f(g)$. By Case 3, each $f_{g}^{\prime}$ is a character of $B$. From the proof of Case 2, we conclude that $f(g b)=$ $f(g) f(b)$ for $g \in G, b \in B$. But, $B$ was an arbitrary Borel subgroup of $G$, and every element of $G$ lies in some Borel subgroup of $G$ [H, Theorem 22.2], so we conclude that $f$ is a character of $G$.

\section{REFERENCES}

[H] J. E. Humphreys, Linear algebraic groups, Springer-Verlag, New York, 1975.

[R] M. Rosenlicht, Toroidal algebraic groups, Proc. Amer. Math. Soc. 12 (1961), 984-988.

Department of Mathematics and Statistics, Memorial University of Newfoundland, St. John's, Newfoundland, CANADA A1B 3X7

Current address: Department of Mathematics, University of Wisconsin-Madison, Madison, Wisconsin 53708 